\title{
Self-powered wireless sensor system application for cutting process control
}

\author{
V. Ostaševičius*, V. Jūrẻnas**, P. Karpavičius***, J. Baskutienė**** \\ *Institute of Mechatronics, Kaunas University of Technology, Kaunas, LT-44244, Lithuania, \\ E-mail: vytautas.ostasevicius@ktu.lt \\ **Institute of Mechatronics, Kaunas University of Technology, Kaunas, LT-44244, Lithuania, \\ E-mail:vytautas.jurenas@ktu.lt \\ ***Institute of Mechatronics, Kaunas University of Technology, Kaunas, LT-44244, Lithuania, \\ E-mail: paulius.karpavicius@ktu.edu \\ ****Department of Production Engineering, Kaunas University of Technology, Kaunas, LT-44244, Lithuania, \\ E-mail: jolanta.baskutiene@ktu.lt
}

cross $^{\text {ref }}$ http://dx.doi.org/10.5755/j01.mech.23.3.17957

\section{Introduction}

The end milling process is used widely in airframe component machining and in die sinking applications, because of its suitability for machining complex shapes and hard-to-reach surfaces.

End milling process can be described as an interrupted cutting process because during each revolution of the cutter, its teeth enter and exit the work piece and in this way, they are subjected to an impact force cycle (Fig. 1).

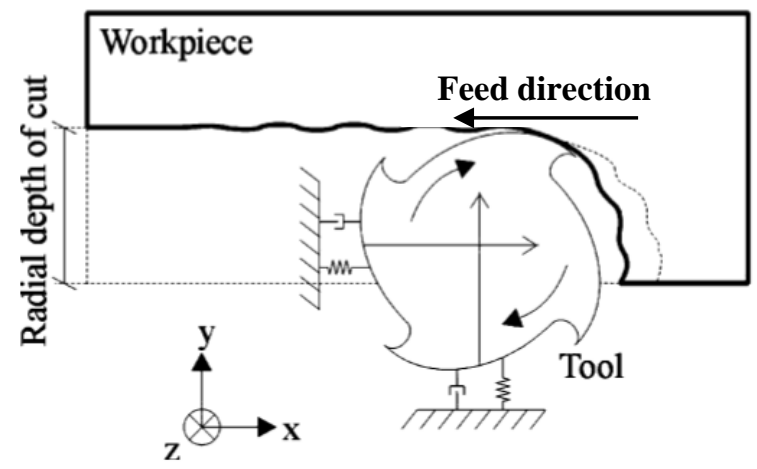

Fig. 1 Schematic representation of the end milling process

In paper [1], the effect of high-frequency excitation of the cutting tool in end milling of blanks from hard metal alloys is presented. It is shown that high-frequency oscillations superimposed on the continuous movement of the tool lead to the stabilization of the milling process with an excellent surface quality compared to conventional machining. The model of finite elements of a vibrating milling tool was developed and tested experimentally. Paper [2] presents an experimental and numerical study of the process of face milling. The purpose of this study is to determine the force acting on one cutting insert. In addition, the final element model of the grinding process was compiled by adopting the hypothesis of a truncated cross section. Finally, finite element simulations were performed to determine the distribution of residual stresses over the depth of the surface being treated.

An attachable wireless vibration module, powered by the energy harvester, to monitor the milling process is demonstrated in [3]. The system consists of an electromagnetic energy harvester, MEMS accelerometer and wireless module. Thanks to the integration of the harvester, accelerometer and wireless module, an autonomous wireless vibration detection system is achieved. The result of testing the system, controlling the milling process, shows that the system successfully recognizes the vibration generated by milling and then transmits vibrating signals to the terminal. The system can demonstrate monitoring of the milling process in real time. To test the system [4], a CNC-machining tool with various milling processes is used. According to the test results, the system is completely autonomous and is capable of successfully perceiving vibrations in milling processes. In addition, by analyzing vibration signals (i.e., by analyzing the electrical outputs of the accelerometers), the criteria are successfully established for the system to accurately simulate the milling processes and cutting conditions in real time (for example, the wear conditions of the cutter and the saw breaker appearance). Study [5] provides an overview of modern sensor technology and its application in the milling process for measuring the processing signal for tool monitoring systems. Processing signals such as cutting force, torque, vibration, acoustic emission, current/power, sound and temperature during milling are briefly considered to determine the process parameters.

Knowledge of the standard operating time of technological operations is an important element in the business operations of each manufacturing enterprise. This is the basis for establishing production capacity, scheduling the production schedule or even costing. The article [6] presents various methods used in calculating the standard operating time of technological operations. Given the growing demands of society, it can be concluded that the main challenges facing modern industry are improving accuracy and productivity, reducing costs and saving in material and energy resources. Increasing productivity, as one of these goals, is especially important for milling due to limitations in the ability to handle complex surfaces. One of the features of the milling process, which directly affects productivity, is the processing strategy, which is the shape of the movement of the tool path during machining. In paper [7], the influence of milling strategies on the roughness of the treated surface is analyzed on the example of a workpiece of complex geometry.

The development of reliable high-speed spindles 
and motion control systems led to an increase in the industrial use of high-speed milling [8]. One of the main applications of this new technology is the manufacture of thinwalled aluminum parts for aircraft. Flexibility of tools and workpieces, high spindle frequencies and inherent nonlinearities of the impact during milling can lead to complex dynamic interactions between the tool and workpiece. An experiment was developed to investigate the vibrations of a thin-walled part during milling. Time series, power spectra, autocorrelations, auto-scenes and phase portraits were investigated. From these data, it follows that the nonlinearity of stiffness and damping, caused by intermittent cutting action, has a pronounced effect on the dynamics of the workpiece. These results are important for the prediction and control of vibrations during milling. Milling of large thinwalled casings with complex features requires appropriate damping solutions to achieve the required quality of the workpiece surface with aggressive processing parameters. Paper [9] presents a new surface damping solution consisting of a thin flexible layer installed with distributed discrete masses attached to the viscoelastic layer. The damping of higher frequencies through the flexible layer and subsequent lower frequencies due to the added masses makes it possible to attenuate in a wide range. A significant amount of research has focused on the dynamics of machining due to the impact it has on productivity and quality [10]. Models were developed with increasing accuracy to predict the dynamic of cutting tool's behavior under various circumstances. However, the behavior of the workpiece during machining is also a current limiting factor that is considered by restricting product designers using functions with subtle characteristics. Numerical and experimental methods were used to obtain stable regions during machining of thin-walled structures, which gives a good comparison [11]. Examples are given and compared with experimental results with a satisfactory agreement.

The novelty of this work is to offer an autonomous wireless technique for controlling the milling process. Research in this area has high expectations in the context of increasing productivity, improving the quality of parts and reducing costs. The success of automation of production processes depends first on the effectiveness of the systems of control and management of the technological process. Key issues to be presented include sensor technology, control technology, equipment availability and an implementation example. The advantage of the proposed methodology and the reason for its implementation lie in the field of industrial application.

\section{Experimental setup used for milling investigation}

To investigate the possibility of harvesting vibrational energy present during milling operation an experiment has been setup (Fig. 2). During experimentation Deckel Maho DMU 35 m CNC universal machining center with five teeth milling tool has been used and on the side of the milling tool a wireless accelerometer was fixed. During milling operation, the accelerometer would register vibrations generated in the tool during its tooth's contact with the machined workpiece the information representing cutters vibrations were transmitted wirelessly to a receiver placed outside the machining center. Information from the receiver was transmitted to personal computer where a specialized information processing program performed required data transformation and visualization of the results. Vibrations registered by the accelerometer are represented as voltage over time. Part of the visualized processed data is presented in Fig. 3.

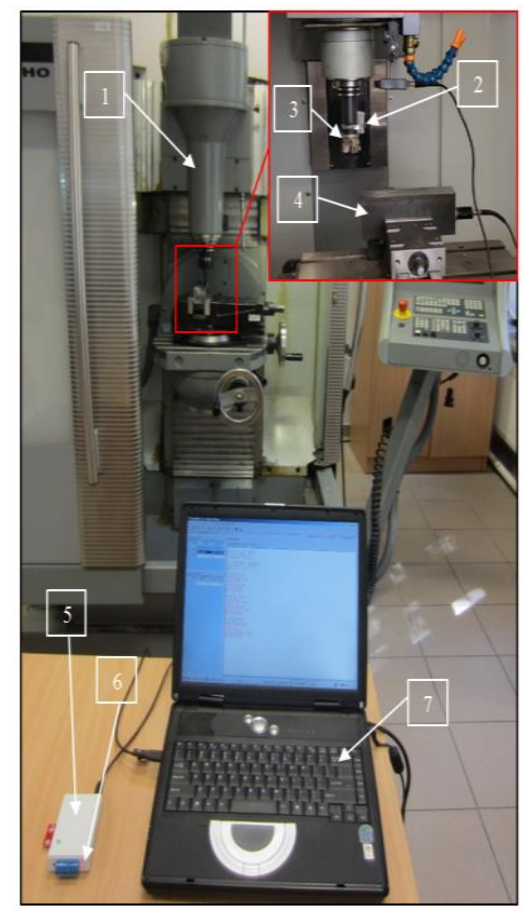

Fig. 2 Deckel Maho DMU 35 m CNC universal machining center experimental setup - global view: 1 - spindle; 2 - wireless accelerometer; 3 - milling tool; 4 - work piece; 5 - receiver; 6 - LED for indication of the critical cutting tool wear; 7 - PC with information processing program

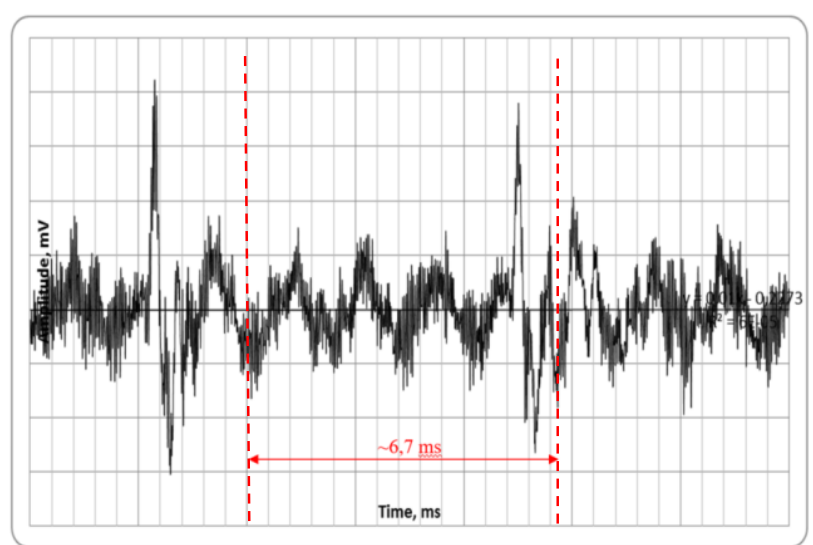

Fig. 3 Face milling tool vibrations at 3200 RPM of 4-tooth mill

Information presented in Fig. 3 shows that the $6.7 \mathrm{~ms}$ period coinciding with the $150 \mathrm{~Hz}$ frequency is dominant as the result of mill tooth and work piece material collision. Having in mind that tool rotation at $3200 \mathrm{rpm}$ provokes approximately $100 \mathrm{~g}$ eccentric force, the mass of selfpowered wireless device strongly influences the dysbalanced of the rotating tool, thus it is necessary to find an appropriate type of the actuator capable to harvest enough amount of energy for the wireless device to operate. A cantilever type piezoelectric transducer is suitable for this type of application. As the piezoelectric cantilever fixed at one of its ends experiences force resulting from any changes in 
acceleration or motion it undergoes mechanical deformations in the direction of the applied force and thus generated an electrical charge at its surface.

For our investigation of milling tool vibrations present during operation a tri-morph d31 type piezoceramic cantilever beam M1100 produced by "Johnson Matthey" has been chosen to be used. The first eigenfrequency for the piezoelectric cantilever beam was simulated using COMSOL Multiphysics software to be equal to 203.72 [Hz] (Fig. 4). At this close to the resonant frequency generated by rotating milling tool is expected to get the higher power output from the piezoceramic element.



Fig. 4 Simulated first eigenfrequency (natural frequency) for M1100 piezoceramic cantilever beam

Investigation of the piezoelectric cantilevers response to excitation over different frequency has been performed. Simulation results showed that as expected the peak voltage generated is around the natural frequency of the cantilever beam $(203 \mathrm{~Hz})$, whose magnitude was evaluated to be 3.1 [V] (Fig. 5) which is sufficient to power harvester's electronics.

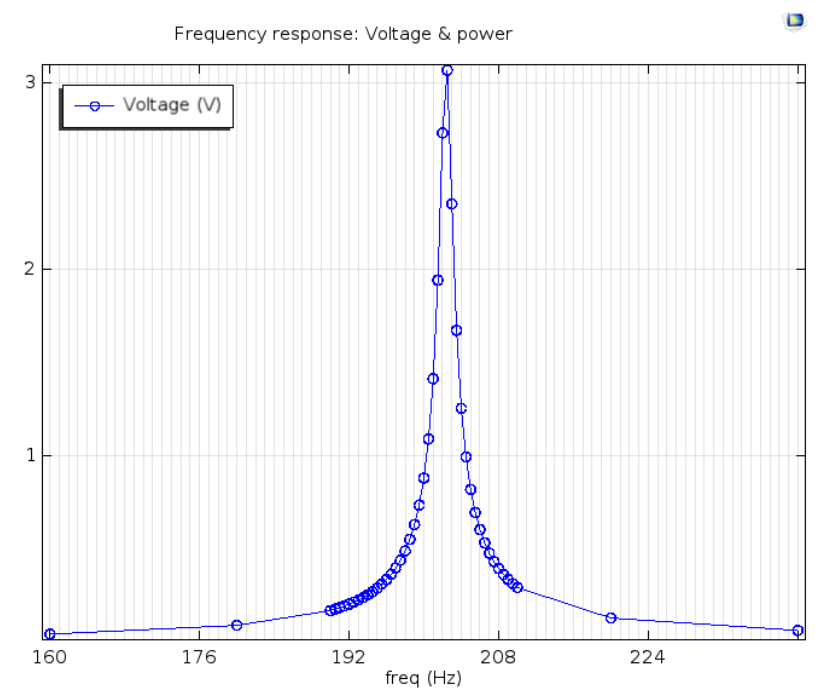

Fig. 5 Voltage generated by M1100 piezoceramic cantilever at its first eigenfrequency

For an energy harvesting and storing system it is required to have energy generating, changing, transforming, stabilizing and storing elements, as well components for protection from overvoltage. Structural scheme of such designed system is shown in Fig. 6.

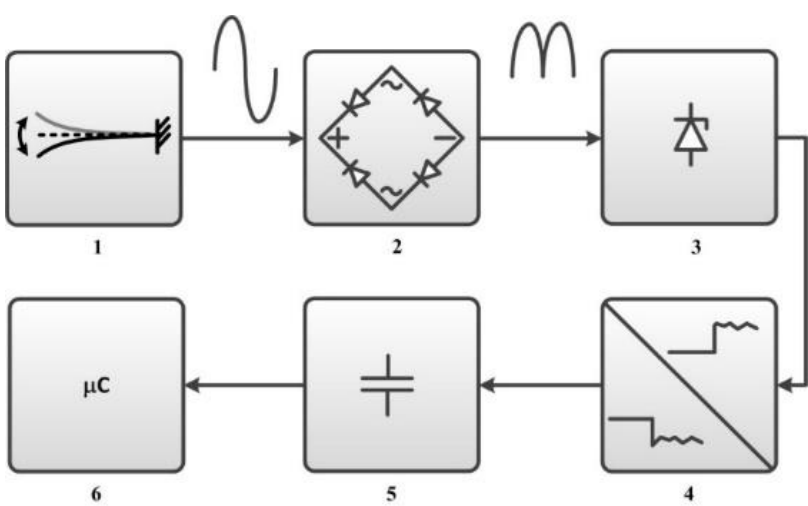

Fig. 6 Components of energy generating and storing elements: 1 - energy transducer; 2 - diode bridge rectifier; 3 - guard element; 4 - voltage conversion and stabilization chip; 5 -energy storage unit; 6 - powered electronics

In general case energy transducer or energy harvesting element is any energy transducer which transforms mechanical energy into electrical. At relatively low frequencies (few hundred $\mathrm{Hz}$ ) all energy transducers have highest energy output when excited at their resonance frequency, this way amount of generated energy has potential to be increased from 2 to 100 times as compared to energy output while working at any other frequency.

Based on observed experimental and simulation results a prototype electrical energy harvester device has been constructed (Fig. 7).

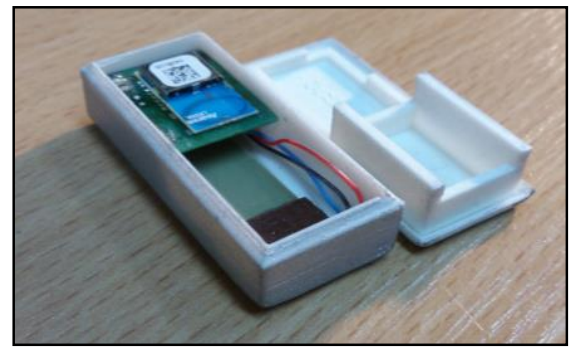

Fig. 7 General view of the electrical energy harvester: 1 - case; 2 - cover; 3 - piezoelectric cantilever; 4 - controller with wireless transmitter

The prototype device consists of a piezoelectric d31 type piezoelectric cantilever beam with a proof mass (Fig. 8) at the free end added to optimize its natural frequency response so that it corresponds to the frequency of excitation vibrations that present during milling operation. Electrical and control circuit that converts generated voltage and transmits wireless signal representing tool vibrations has been developed and incorporated into the prototype device.

In order to achieve highest energy generation efficiency and sensitivity to vibrations of the device, piezoelectric sensor-transducer's resonance frequency of 1 bending moment $F_{k}(t)$, with the application of 2 a proof mass, is synchronized so that is would be as near as possible to the vibrational frequency excited by the cutting forces $F_{p}(t)$. For the device, a "Johnson Matthey" M1100 type tri-morph piezoelectric transducer has been used, whose natural frequency response with the application of a 2 proof mass can be adjusted from $203 \mathrm{~Hz}(0 \mathrm{~g}$ proof mass) to $91 \mathrm{~Hz}(1.2 \mathrm{~g}$ proof mass) (Fig. 9). 


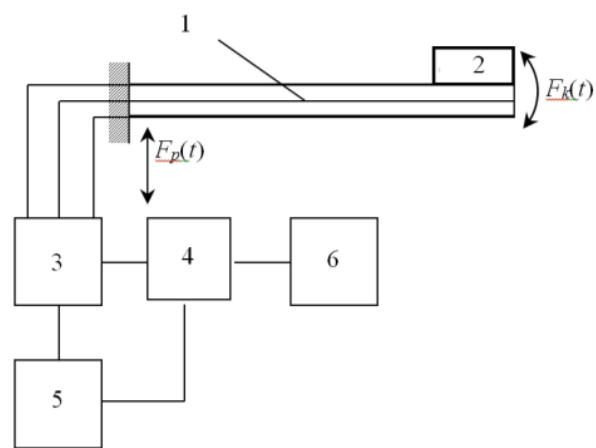

Fig. 8 Structural scheme of vibrational sensor with integrated energy harvester and wireless signal transmitting unit: 1 - piezoelectric vibrational energy sensortransducer; 2 - proof-mass used for initial adjustment of the piezoelectric vibrational energy sensor-transducer's natural frequency; 3 -generated voltage's conversion unit; 4 - control unit; 5 - energy storage unit; 6 - wireless signal transmitting unit

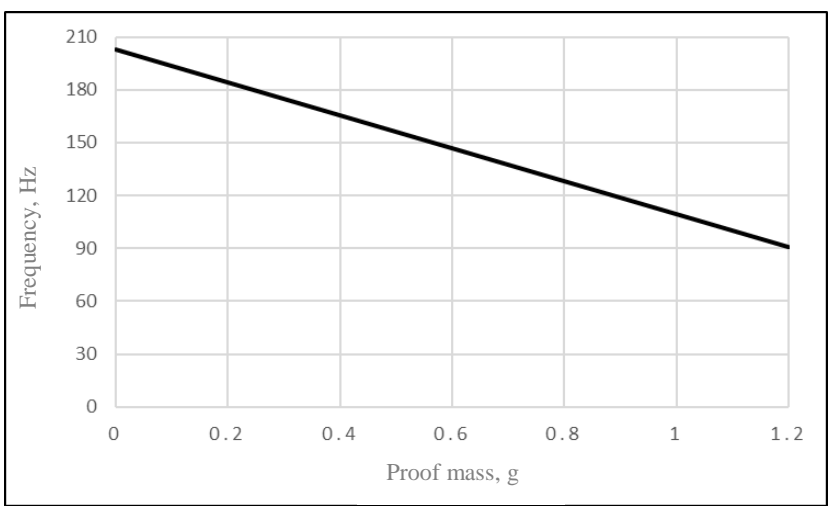

Fig. 9 "Johnson Matthey" M1 100 piezoelectric transducer's natural frequency dependence on the proof mass

Since this this type piezoelectric transducer is composed of two piezoelectric material layers (tri-morph type) it's resonance frequency can be adjusted in between of $10 \mathrm{~Hz}$ limit by connecting to one of the layers some load shunt resistor and the other piezoelectric layer is used to measure vibrations and electrical energy generation level. Fig. 10 shows two amplitude-frequency characteristics (at

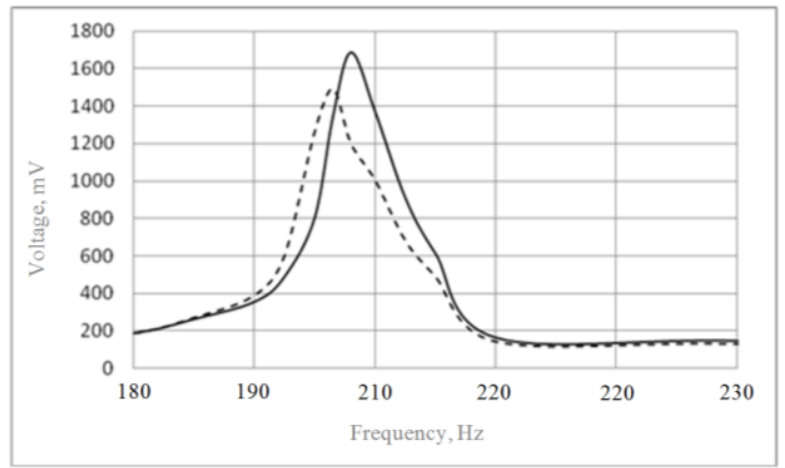

Fig. 10 Piezoelectric transducers generated electrical signal amplitude-frequency characteristics: ---- - line, when one of the piezoelectric layer is connected in short circuit; — - line when one of the piezoelectric layer connection is open transducers resonance frequency), when one of the characteristics (solid line) represents when the resistor value tends to infinity, that is one of the piezoelectric layer's contacts are open, and the other one (dashed line), when the resistor value is equal to zero - when one of the piezoelectric layer is connected in short circuit.

This type of piezoelectric transducer's resonance frequency control allows to wirelessly fine control any small change in vibration excitation frequency, that appears during machining operation.

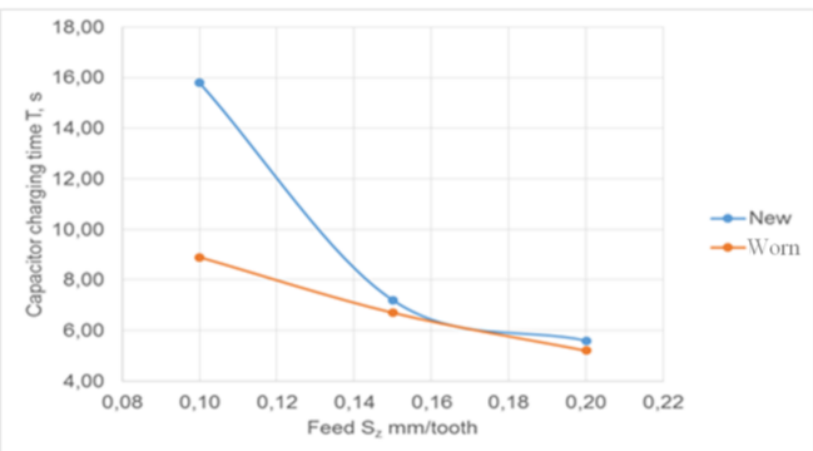

Fig. 11 Charging time and feed relation graphs when spindle speed is set $n=1000 \mathrm{rpm}$ and cutting depth $a_{p}=2 \mathrm{~mm}$

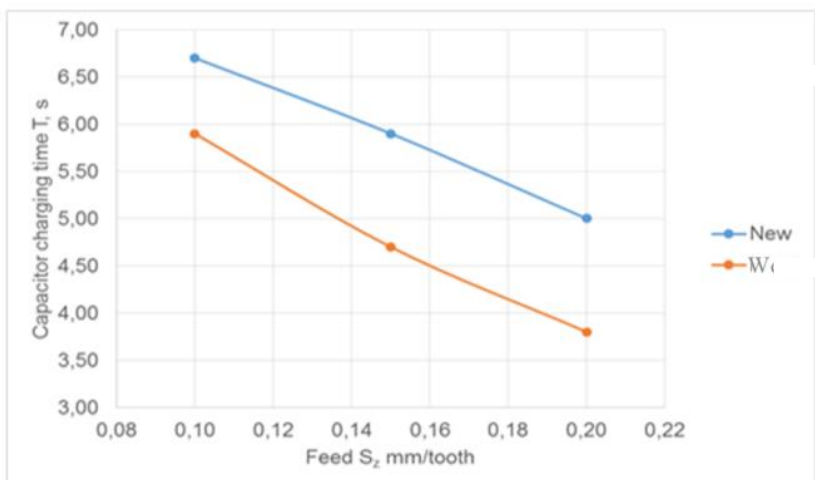

Fig. 12 Charging time and feed relation graphs when spindle speed is set $n=1000 \mathrm{rpm}$ and cutting depth $a_{p}=3 \mathrm{~mm}$

Experiments were performed on the developed prototype. During milling operation, different spindle rotation speed $n$, feed $S z$ and cutting depth ap. has been set to evaluate the devices response. The device is excited by vibrations present during cutters tooth contact with the workpiece, electrical charge generated by the piezoelectric cantilever is transferred to power its electronics where signal representing tool vibrations is created and wirelessly sent to receiving unit outside the milling machine for further processing and evaluation. The signal sent by the electrical energy harvester is generated by charging a capacitor, which charging time depends on the amplitude and frequency of charge generated by the piezoelectric cantilever beam during machining process. As the tool tends to wear the amplitude of the vibrations generated during tool-workpiece contact increases, thus consequently the amount of charge created by the piezoelectric cantilever increases, leading to increase in voltage supplied to the devices circuit that reduces the capacitors charging time. Experimental steel workpiece processing results are presented in Figs. 11 and 12 where capacitors charging time is represented and compared for 
new and worn tool under different machining conditions.

As expected for the same machining conditions the charging time of capacitor for worn out tool is lower than for new tool due to increased amplitude of vibrations created at the tool-workpiece contact during machining. Once the tool over certain amount of time starts to degrade, vibrations created during cutter-workpiece interface tend to have higher amplitudes what in turn results in higher power generation by piezoelectric transducer and more electrical energy supplied to the capacitor, decreasing its charging time.

The change in frequency of capacitor discharge time can be interpreted as representing the milling tools condition and its degradation over time during machining.

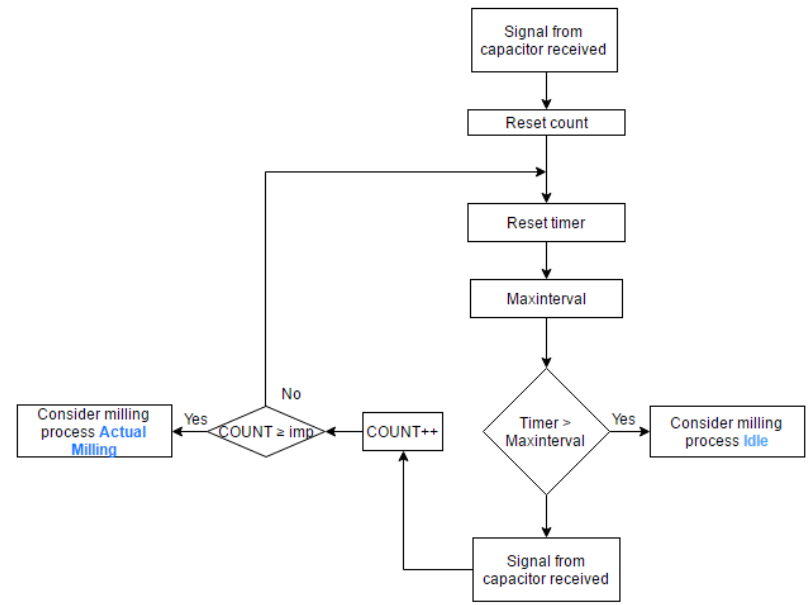

Fig. 13 Control flow chart for evaluation of Actual/Idle milling conditions

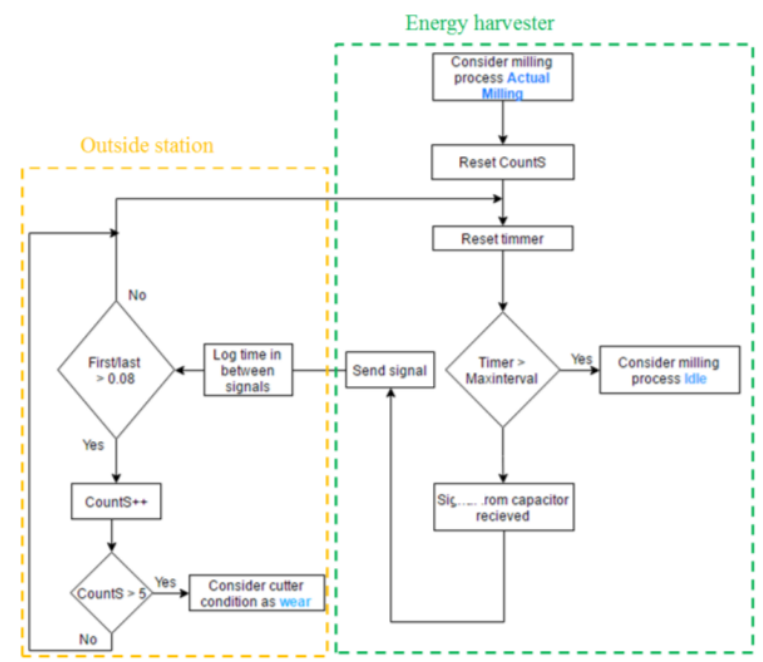

Fig. 14 Control flow chart evaluation of cutter tool wear

Control algorithm, in the form of a flow chart, is proposed for the milling process control in Fig. 13 and Fig. 14. The control algorithm is proposed so that proposed device works only during operation of the milling machine, when the energy generated by piezoelectric transducer powers electronics that convert, manipulate and transmit tool vibration data in the form of interrupted wireless signals. In Fig. 13 is presented scheme to wake up the device and evaluate if the received signal to MCU represents actual milling conditions (that the machine is working instead of excitation provided from outside sources such as during maintenance of the machine). As the first signal (representing milling operation) received resets signal counter "count" and clock "timer" of the MCU. "Maxinterval" must be set in advance and it represents the value of maximum time required/expected for the capacitor to fully charge during milling operation (this time can depend on the set milling operation conditions and the machined workpiece material). As the first, signal wakes up the device, subsequent signal is used to evaluate if capacitor discharge has been generated by actual milling and not by outside interference (such as maintenance or other factors). Number of signals required to evaluate the operation as "Actual Milling" must be set prior for variable "imp", if the number of signals received is higher than "imp", the operation is evaluated as "Actual milling" and device goes into working mode (it is concluded that actual milling operation of some workpiece is undergoing), if any of the signals are delayed for more than set "Maxinterval", the operation is assumed as "Idle" and the MCU goes to "sleep" mode and waits for excitation to start the whole process again. Once the state is evaluated as "Actual Milling" all subsequent signals are transmitted wirelessly.

Second part of the control algorithm is proposed for the evaluation of the cutter tool's condition when operation is evaluated as "Actual Milling" (Fig. 14).

It is separated into two parts, one (right side) for energy harvester and its response once operation is evaluated as actual milling and the second (left side) for evaluation of the cutters condition if it has started to degrade. If the signal from discharging capacitor is received it is sent wirelessly to an outside workstation for evaluation of cutter's tool condition, if it is delayed longer than set "Maxinterval" the device goes to "sleep" mode ("Idle") where it waits for excitation and repeats the cycle for evaluation of actual milling operation. Once the signal is sent to an outside workstation, it is evaluated for indication of cutter tool wear condition. Here the time intervals in between signals are logged, if the difference between first time interval and the one before it is more than $8 \%$ (based on information from Fig. 12) for more than five subsequent times (the number of signals for tool condition evaluation has to be adjusted based on further investigation), the tool condition is evaluated as "wear" and the system or machine operator is informed that tool condition is started to degrade. Further investigation has to be done in order to define when the tool condition could be defined as critical or such that significantly affects the surface quality of the machined workpiece based on change (increase) in period between subsequent signals.

The device's ability to accurately measure milling tool's impact-dynamics during operation enables it to be used to improve surface quality of the workpiece that is machined during high-speed milling of thin-walled structures, where effects off impact dynamics are dominant. The inherent low stiffness of thin wall geometries during high-speed milling leads to generation of regenerative chatter in the workpiece-tool interface that causes poor surface quality and can lead to breakage of the milling tool. Chatter growth leads to fast increase in the amplitude of the impact vibrations present at the tool-workpiece interface. Application of proposed wireless sensor node, can enable detection of chatter presence and growth. The principle to be used for chatter vibration measurement is like the one used for detection of milling tool wear, where increase in accelerations exerted on the device that are driven by higher workpiece-tooth impact forces due to wear of the cutter, causes increase in energy generation that can be related to condition of the tool. Since chatter growth time is significantly higher than the 
cutter's degradation time, increase in amount of energy generation by the device over short time period represented by increased capacitor discharge frequency can be used for prediction of regenerative chatter. This information of chatter and its magnitude can be fed back to the milling machine for control of machining parameter to mitigate this undesirable effect since appearance of chatter and its amplitude depend on milling tools speed of rotation, feed rate and feed depth. Further investigation and experimental work is needed to confirm the applicability of the proposed piezoelectric device for chatter measurement, though results from current investigation during milling provides high expectations for the devices applicability for chatter present during highspeed milling operation of thin-walled parts detection and control.

\section{Acknowledgements}

This work was funded by EU Structural Funds project program "High level R\&D": measure 01.2.2-LMT-K718.

\section{References}

1. Ostasevicius, V.; Gaidys, R.; Dauksevicius, R.; Mikuckyte, S. 2013. Study of vibration milling for improving surface finish of difficult-to-cut materials, Stroinski Vestnik-Journal of Mechanical Engineering, 59(6): 351-357. http://dx.doi.org/10.5545/sv-jme.2012.856.

2. Gyliene, V.; Ostasevicius, V. 2012. Modeling and simulation of a chip load acting on a single milling tool insert, Stroinski Vestnik-Journal of Mechanical Engineering 58(12): 716-723. http://dx.doi.org/10.5545/sv-jme.2012.356.

3. Chung, T-K.; Lee, L.; Tseng, C-T.; Lo, W-T.; Wang, C-H.; Wang, W-C.; Tu, C-T.; Tasi, P-Y. and Wen Chang, J-W. 2013. Self-powered wireless vibrationsensing system for machining monitoring, Proc. of SPIE: 8692 86922U-1, 6.

4. Chung,T-K.; Yeh, P-C.; Hao Lee H.; Lin, C-M.; Tseng C-Y.; Lo, W-T.; Wang, C-M.; Wang, W-C.; Tu, C-J.; Tasi, P-Y. and Chang, J-W. 2016. An attachable electromagnetic energy harvester driven wireless sensing system demonstrating milling-processes and cutter-wear/breakage-condition monitoring, Sensors: 16(3): 269, 18.

5. Rizal, M.; Ghani, J.A.; Nuawi, M.Z. and Haron, C.H.C. 2014. A Review of sensor system and application in milling process for tool condition monitoring. Research J. of Applied Sciences, Engineering and Technology: 7(10): 2083-2097. http://dx.doi.org/10.19026/rjaset.7.502.

6. Skoczylas, L.; Skoczylas, K.; Wydrzyński, D. 2016. The standard milling time of flat surfaces without outline restrictions, Advances in Science and Technology Research J. 10(31): 229-234. http://dx.doi.org/10.12913/22998624/64061.

7. Mlađenović, C.; Kalentić, N.; Zeljković, M.; Tabaković, S. 2014. The influence of milling strategies on roughness of complex surfaces, J. of Production Engieering 17(1): 51-54.
8. Davies, M.A.; Balachandran, B. 2000. Impact dynamics in milling of thin-walled structures, Non-linear Dynamics 22(4): 375-392. http://dx.doi.org/10.1023/A:1008364405411.

9. Kolluru, K.; Axinte, D.; Becker, A. 2013. A solution for minimizing vibrations in milling of thin walled casings by applying dampers to workpiece surface, CIRP Annals - Manufacturing Technology 62(1): 415-418.

10. Mundim, R.B.; Borille, A. V. 2017. An approach for reducing undesired vibrations in milling of low rigidity structures, The International Journal of Advanced Manufacturing Technology 88(1): 971-983. http://dx.doi.org/10.1007/s00170-016-8804-0.

11. Adetoro, O.B.; Wen, P.H.; Sim, W.M; Vepa, R. 2009. Stability lobes prediction in thin wall machining, Proceedings of the World Congress on Engineering: I WCE London, U.K.

V. Ostaševičius, V. Jūrėnas, P. Karpavičius, J. Baskutienė

SELF-POWERED WIRELESS SENSOR SYSTEM APPLICATION FOR CUTTING PROCESS CONTROL

S u m m a r y

In the article a self-powered wireless sensor used for milling operation control is presented. Source of vibrations suitable for energy harvesting and generation has been accurately identified, experimental work on CNC machine performed and was determined that the highest amplitude vibrations for the milling tool are present during its tooth contact with the workpiece. During investigation, it was determined that based on feed rate and number of teeth on the cutter, the frequency of tooth contact with workpiece is in the range of $150-300 \mathrm{~Hz}$, based on that a piezoelectric cantilever with natural frequency falling within this frequency range scope has been chosen. In order to increase the efficiency of energy generation by the piezoelectric transducer adjustment of its natural frequency by adding proof mass for course control and connection of piezoelectric layers for fine tuning has been proposed. Proposed energy generation, storage and wireless signal representing tool condition transmission circuit for the device. Experiments were performed, that allowed to record milling tool's wear during machining and evaluate energy generation dependence on set milling parameters. Designed and validated prototype device is suitable for control of milling process and can be effectively used to increase milling accuracy of thin-walled workpieces.

Keywords: piezoelectric cantilever, tool vibrations, charging time.

Received April 14, 2017 Accepted June 08, 2017 